\title{
Agnes von Wyl, Volker Tschuschke, Aureliano Crameri, Margit Koemeda-Lutz und Peter Schulthess (Hrsg.): Was wirkt in der Psychotherapie? Ergebnisse der «Praxisstudie ambulante Psychotherapie» zu zehn unterschiedlichen Verfahren (Psychosozial, Giessen, 2016)
}

\author{
Marie-Luise Hermann (Zürich)
}

Der in der Reihe «Forschung Psychosozial» herausgegebene schlanke Band dokumentiert die «Praxisstudie ambulante Psychotherapie Schweiz» (PAP-S), ein Forschungsprojekt der Schweizer Charta für Psychotherapie in Kooperation mit dem Klinikum der Universität zu Köln und dem Departement Angewandte Psychologie der Zürcher Hochschule für Angewandte Wissenschaften ZHAW. Die Charta als Verband verschiedener Psychotherapieausbildungsinstitute und Fachverbände hat sich 2002 in einer Deklaration dazu verpflichtet, wissenschaftliche Wirksamkeitsnachweise ihrer Psychotherapiemethoden zu erbringen. In Zusammenarbeit mit Volker Tschuschke (Köln) wurde dafür ein «naturalistisches Prozess-Outcome-Studiendesign» entwickelt, das an der ZHAW (Leitung Hugo Grünwald, später Agnes von Wyl) durchgeführt wurde.

Das Besondere an dieser umfassenden Studie liegt in einem Design, das die vielfältige ambulante psychotherapeutische Praxis in der Schweiz möglichst nah abzubilden versucht und in einer Kombination von Untersuchungsmethoden ("multi-method": Fragebögen, Audiodateien aller Therapiestunden) und Informanten ("multi-informant": Patient/innen, Therapeut/innen) sowohl den Therapieprozess als auch die Ergebnisqualität (“Outcome”: Drei Messzeitpunkte Prä, Post und Ein-Jahres-Katamnese) verfolgt. Zudem sollte das Design zehn verschiedene Therapieverfahren auf die zentrale Frage hin erfassen: «Was wirkt in der Psychotherapie?»

Dass Psychotherapie wirkt, ist unter wirtschaftlichem gesundheitspolitischem Druck in Tausenden von internationalen Studien belegt worden. Dafür wurden aus der evidenzbasierten medizinischen Forschung übernommene RCTStudien (randomized controlled trials) zum «Gold-Standard» von Studiendesigns erklärt. Zahllose "horse-race"-Vergleiche grosser Therapieschulen, meist kognitive Verhaltenstherapie vs. psychodynamische Kurz- vs. Langzeittherapie, zeigten eine 
vergleichbare Wirksamkeit für alle Störungsbilder (aktuelle Übersicht in Rabung \& Leichsenring, 2016). Diese unter Laborbedingungen entstandenen Resultate zur «Wirksamkeit» ("efficacy studies") haben jedoch wenig mit der naturalistischen Praxis der Psychotherapie und ihrer «Effektivität»zu tun ("effectiveness studies”), wie dies die PAP-S-Studie angestrebt hat, um für weitere Psychotherapie-Schulen der Charta ihre Wirksamkeit nachzuweisen.

In zwölf Kapiteln stellen die Autor/innen den gesamten Prozess der von 2006 bis 2012 dauernden Datenerhebung bis zum Abschluss der Auswertungen 2015 mit Ergebnissen dar. Nach der Vorgeschichte der Studie (Kap. 1) wird die Kooperation zwischen Charta und den beiden Hochschulen sowie die Wahl des naturalistischen Prozess-Ergebnis-Designs mit detaillierter Kritik an den RCT-Studien begründet (Kap. 2). Das in Kapitel 3 erläuterte Studiendesign soll hier kurz vorgestellt werden: Von 86 Therapeut/innen aus neun verschiedenen Charta-Instituten (humanistische, tiefenpsychologische sowie körper- und kunstorientierte Psychotherapien) sowie einzelnen psychoanalytischen Psychotherapeut/innen nahmen 362 Patient/innen zwischen 17 und 72 Jahren an der Studie teil. An drei Untersuchungszeitpunkten erhoben geschulte Psychotherapeut/innen u. a. Symptom-Fragebögen, das psychiatrische SKID-I und II-Interview sowie die Konflikt- und Struktur-Achse der OPDDiagnostik. Zur Prozessmessung schätzten nach jeder fünften Sitzung Patient/ innen und Therapeut/innen die Qualität der Arbeitsbeziehung ein, die Therapeut/ innen auch ihre angewendeten Interventionen. Anhand der Audiodateien wurde die Konzepttreue des Therapieverfahrens beurteilt.

In den weiteren Kapiteln werden diese Instrumente und Ergebnisse anhand von Tabellen, Grafiken oder Beispielen anschaulich erläutert und kritisch diskutiert: So wird das Engagement im jahrelangen Schulungsprozess für das OPD-Rating aufgezeigt (Kap. 4) und die zeitaufwendige Einschätzung von Interventionstechniken mithilfe eines eigens für die Studie entwickelten Rating-Manuals (Kap. 5). Kapitel 6 informiert prägnant über den aktuellen Stand zu Wirkfaktoren in der Psychotherapieforschung, die zu den zentralen Befunden der Studie führen (Kap. 7): Die Kategorisierung der therapeutischen Interventionen zeigt, dass lediglich zwischen 5-28\% auf spezifische Wirkfaktoren fallen (d.h. Konzepttreue zur jeweiligen Therapieschule), während nichtspezifische Interventionen 50-73\% ausmachen und $18-27 \%$ fremdschulische Interventionen angewendet wurden. Das in der Forschung häufig beschriebene Äquivalenzparadoxon, dass trotz verschiedenster Behandlungskonzepte verschiedene Verfahren ähnlich wirksam sind, wird auch hier bestätigt und dahingehend interpretiert, dass erfahrene Psychotherapeut/ innen ihr Behandlungsrepertoire im Lauf der Zeit über das erlernte Grundverfahren hinaus erweitern und es auf ihre Patient/innen anpassen. Die Frage «Was wirkt in der Psychotherapie?» kann daher nur mit dem Verweis auf sehr viele Variablen (v. a. auf Seiten der Patient/innen, ihrer Störungen und Belastungen) in einem «hochkomplexen zwischenmenschlichen Prozess» beantwortet werden (S. 83).

Die Qualität der therapeutischen Beziehung (Kap. 8) als zentralem unspezifischem Wirkfaktor erwies sich bereits in den ersten fünf Sitzungen als signifikanter Prädiktor des Behandlungserfolgs, belastete Arbeitsbeziehungen waren vor allem auf chronische und strukturelle Störungen und nicht die Symptombelastung zurückzuführen. Im Nachweis der Wirksamkeit über OutcomeErgebnisse (Reduktion der Symptombelastung und strukturelle Veränderungen) wurden vergleichbare Effektstärken wie in evidenzbasierten Studien der kognitiven Verhaltenstherapie erreicht (Kap. 9). Weitere Kapitel präsentieren Ergebnisse zu therapeutischen Techniken sowie Unterschiede zwischen Therapeut/innen sowie Geschlechts- und Gender-Aspekte.

Angesichts des herausfordernd komplexen Studiendesigns und einer enormen Datenfülle ist es den Autor/innen hervorragend gelungen, eine gut lesbare, überblicksartige Zusammenfassung des gesamten Projektes, der Forschungsinstrumente sowie der Einzelbefunde zu gestalten. Wer sich noch nicht mit Psychotherapieforschung befasst hat oder in Ausbildung ist, wird in wichtige Forschungsdiskussionen auf verständliche Weise eingeführt. Aber auch bereits informierte Leser/innen finden interessante Ergebnisse und Hinweise auf mögliche künftige Fragestellungen für die Praxisforschung. Wer noch mehr wissen möchte, kann die detaillierteren Publikationen der Autor/innen und zahlreiche Literaturhinweise verfolgen. Der wichtige Hinweis, warum gerade diese zehn Therapieschulen einbezogen wurden und weitere Ausbildungsinstitute (wie KVT, systemische, personzentrierte) auf Einladung nicht reagiert hätten (S. 20), könnte hingegen am Ende des Vorworts auch übersehen werden.

Die wissenschaftliche Einordnung des gesamten Projekts wird nachvollziehbar dargestellt, dieWahl der einzelnen Forschungsmethoden oder Fragebögen jedoch nicht durchgängig begründet. Auf den in Aussicht gestellten «reichhaltigen Fundus an Daten», der «noch auf Jahre hinaus Auswertungen, Dissertationen und Veröffentlichungen» ermöglichen solle (S. 28), darf man gespannt bleiben. Zur näheren Untersuchung, was wirkt, wäre dafür auch die Ergänzung um zeitintensive qualitative Einzelfallstudien (vgl. Mathys, Arboleda et al., 2013) sinnvoll und wünschenswert 


\section{Literatur}

Mathys, H., Arboleda, L., Boucsein, V., Frei, M., Hermann, M.-L., Luder, M., Neukom, M. \& Boothe, B. (2013). Alexandra - eine multiperspektivische, qualitative Einzelfallstudie zu Anliegen von PatientInnen im psychodynamischen Erstinterview [75 Absätze]. Forum Qualitative Sozialforschung 14 (2), Art. 20, http://nbn-resolving.de/urn:nbn:de:0114-fqs1302207 [04.03.17].

Rabung, S. \& Leichsenring, F. (2016). Evidenz für psychodynamische Langzeittherapie. Überblick über vorliegende Reviews. Psychotherapeut 61, 441-446. 